EVS27

Barcelona, Spain, November 17-20, 2013

\title{
Development of an in-series hybrid urban bus model and its correlation with on-board testing results.
}

\author{
Daniel Resino Zamora ${ }^{1}$, José María López Martínez, Carlos Julio Loboguerrero Carrasco, \\ Juan José Delgado Vaca \\ ${ }^{1}$ Automotive Research Institute - Technical University of Madrid. \\ Crta. Valencia km7-Campus Sur UPM - 28031 - Madrid-Spain \\ daniel.resino@upm.es
}

\begin{abstract}
New emission standards implemented in EU has resulted in an increasing use of passenger hybrid vehicles as well as hybrid heavy-duty transport vehicles. Manufacturers have been offering a wide range of efficient and lower emission vehicles to fulfil these Regulations. Besides commercial vehicles, it it's also important to consider the use and implementation of hybrid urban buses because it is a massive way of transportation that is used every day in the bigger cities. The use of hybrid urban buses is significant to achieve the goal for lower emissions and better energy efficiency, due to the city utilization involves several stops and accelerations. This paper considers the comparison between the results obtained from software simulation of a complete in-series hybrid urban bus platform (develop under AVL Cruise ${ }^{\circledR}$ software) and the data obtained from real on-board measurements made in the city of Madrid. The model involves an in-series hybrid electric bus, meaning that the propulsion system is supplied by an electric motor which receives energy from a stored source (battery). The analysis of the results shows a very accuracy approach and lower emission vehicle, with an error below $5 \%$ in fuel consumption modelling a real driving cycle up to $8 \mathrm{~km}$.
\end{abstract}

\section{Introduction}

It is known that nowadays the automotive industry is focusing in more efficient vehicles with different propulsion systems such as pure electric vehicles or hybrid electric vehicles. There is a wide range in the passenger vehicle segment with these new technologies but there is also a significant growth in the heavy-duty truck and bus segments. As main European cities are specially focused on improve their air quality, it is important to study not only the passenger vehicle segment but the heavy-duty truck and bus segments, like urban transport buses [6][10].

Nowadays, industry is looking for solutions that make possible to improve the accuracy between design processes and final results; but costs are 
becoming more determining and thus, software simulations are the way to get both goals. [7]

This paper is focused on the modeling and simulation of an in-series hybrid electric urban bus with AVL Cruise ${ }^{\circledR}$ software. The results of the simulation are compared with those obtained in a drive cycle made in the city of Madrid in real conditions with a full size hybrid electric bus.

\section{Objective}

Hybrid electric buses can be used as long range vehicles since they are constantly charging batteries and therefore, reducing emissions compared to conventional models. With the model, it will be possible to achieve a software platform to estimate consumption and emissions along a given drive cycle (i.e. urban bus line) as well as having a tool to develop and compare different control strategies.

The main objective of the project is to compare the efficiency and performance between the drive cycle made with the full size bus and the simulation made with the software. The efficiency will be determined as the strategy method implemented in both systems according to the state of charge of the batteries and the usage of the combustion engine. In the other instance, $\mathrm{CO}_{2}$ emissions from the combustion engine will be evaluated as well.

The comparison will determine the accuracy of the model and its approach to the driving cycle in real conditions.

\section{Configuration of the Hybrid Urban Bus}

As it has been stated before, the configuration of the real size hybrid urban bus consist of an inseries hybrid powertrain. This means that the drive is supplied by an electric motor which receives the energy from a battery or directly from the internal combustion engine through an electric generator. As it can be seen in figure 1, the engine and generator are not mechanically coupled to the power train.

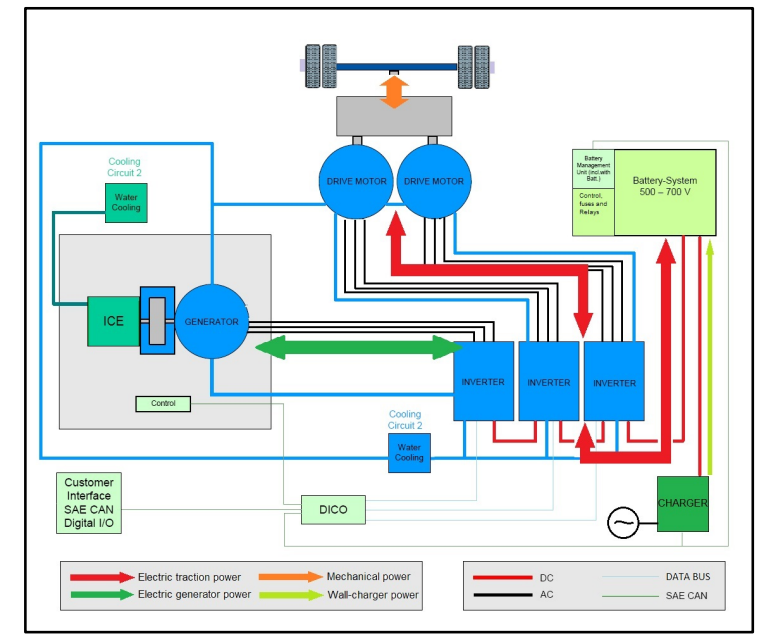

Figure 1: In-series hybrid configuration scheme. Electric, mechanical, thermal and data lines.

The output of the electric generator is connected to a power DC Bus through a controllable electronic converter [8]. This configuration allows the implementation of a bidirectional energy source (battery) which is connected with a DC/DC converter to allow the energy flow in both directions. The electric motor is also connected to the DC power bus to allow its operation either as a traction motor, or as a generator.

The in-series hybrid configuration is derived from pure electric vehicles on which an additional engine-generator is added to the system to extend the operating range that is limited by the energy density of the batteries.

For the proper functioning of the in-series hybrid, it's necessary to employ a controller to achieve the intended low emission and low fuel consumption from the internal combustion engine, as well a long range driving [3][4][5]. The controller is essential to determine the behaviour of the vehicle in many operation modes:

$\checkmark \quad$ Electric traction mode: The electric motor gives propulsion to the vehicle, taking the energy from the battery. The enginegenerator set is turned off.

$\checkmark$ Hybrid mode: The traction power is supplied by the electric motor and the engine-generator set.

$\checkmark \quad$ Traction with battery charging mode: In this process, the engine-generator set is supplying power enough to charge the batteries and to propel the electric motor simultaneously. 
Regenerative braking mode: Regenerative braking is made when the vehicle is slowing down, making the electric motor acting as a generator to store the kinetic energy that the vehicle is generating in the braking process. The engine-generator set is turned off during this mode.

$\checkmark$ Battery charging in hybrid mode: This mode is achieved when the enginegenerator is working and at the same time, when the electric motor operate as a generator (in regenerative braking).

$\checkmark$ Engine start-stop mode: The enginegenerator set is turned off while the vehicle is stopped in any part of the route (bus stop, red light, traffic congestion, etc).

Depending on the driving conditions, the controller will employ any of these modes to achieve a better performance of the vehicle.

\section{Project Stages}

The stages for this project consists in a carefully set of steps divided into model tasks and measurement tasks. All the steps were created to perform a close simulation to the real test [11]. The stages of development are:

\subsection{Creation of Hybrid Urban Bus plant model in the software.}

The model is based in the components used in the real size Bus.

4.1.1 Modules: All data and values from the real size bus were introduced in the corresponding modules.

4.1.2 Controllers: The programming of the controller consists in doing a controller for the regenerative braking, for the electric drive, and for the hybrid mode (range extender). The controllers are activated according to the driving cycle and to the operating modes described in section 3 .

4.1.3 Data bus linking: Data communication between modules is made via a "Data Bus" employed in the software. This allows the exchange of information between the modules and the controllers to allow its operation. It simulates the embedded network system of the vehicle.

\subsection{Definition of simulation conditions.}

This stage consists of the definition of parameters that approaches the simulation to the real test, such as velocity profile, altitude profile, driver behaviour, etc.

\subsection{Simulation run and verification.}

This step is for verifying the driving performance of the vehicle. The operating modes are evaluated in this step according to the driving profile.

\subsection{Real cycle data collecting. equipment and measurements.}

The real cycle data collecting consist in employing the proper instrumentation in the full size hybrid urban bus in order to collect and measure data of the driving behaviour.

\subsection{Results comparison}

A full drive cycle simulation was performed in order to evaluate and compare the results of the simulation and the real test. The results involve the electric motor drive (speed and torque), the mechanical power the electric motor develops, and the current and voltage generated in the driving cycle.

\section{Model}

\subsection{Model plant}

The model built in AVL Cruise ${ }^{\circledR}$ can be seen in figure 2. Vehicle components are arranged by modules, which are mechanically connected by blue lines or electrically connected by red lines.

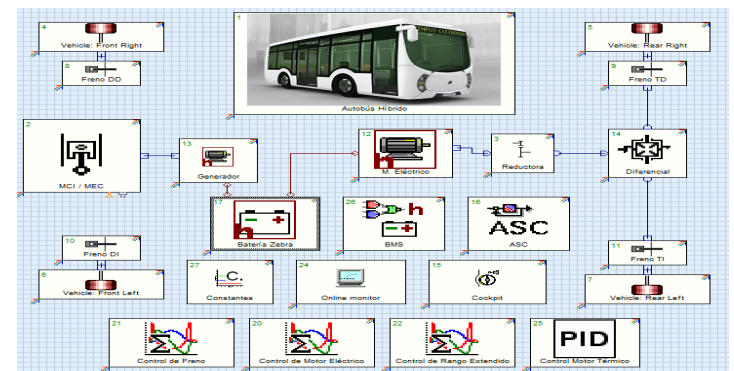

Figure 2: Model plant of Hybrid urban bus built in AVL Cruise ${ }^{\circledR}$ software. 


\subsubsection{Modules}

Model consists of an internal combustion engine mechanically coupled to a generator, which transfers the energy to a battery. The electric motor receives the energy form the battery to supply it to the rear wheels of the bus through a single relation gearbox and a differential.

The rest of components determine the elements of the closest architecture of the real size bus.

Since the software offers a wide range for simulating diverse vehicles, the ECU and data communications between components are established via a "data bus" for proper data communication and transfer between modules [3$5][8][9]$.

\subsubsection{Controllers}

Controllers are built inside the same software using $\mathrm{C}$ code programming. Three controllers were programmed to control all powertrain systems. These three main controllers consist of a brake controller, an electric drive controller and a hybrid mode controller. The first one is to determine when the brake pedal is applied to convert the braking torque in braking pressure for all four brakes; this will generate a negative torque and therefore transfer it to the electric drive motor. The second controller is necessary to allow the electric drive motor to transfer the drive to the wheels and to receive the output data of the first controller. The third controller determines de control strategy for the hybrid. This controller is the responsible for performing an efficient driving cycle.

\subsubsection{Data bus linking}

Data communication between modules in the model, are made through a Data Bus linking. This allows the proper data transfer to command the optimal operation of the vehicle. The Data Bus employed in the software, is equivalent to the real BUS CAN of the vehicle which allows the user to see in a graphical way the links between the modules.

\section{Simulation conditions}

\subsection{Velocity profile}

Thanks to the measured data obtained on board the real size hybrid bus, it is possible to transfer the driving profile to the software, meaning that the simulation will trace the same route as the real size bus did.

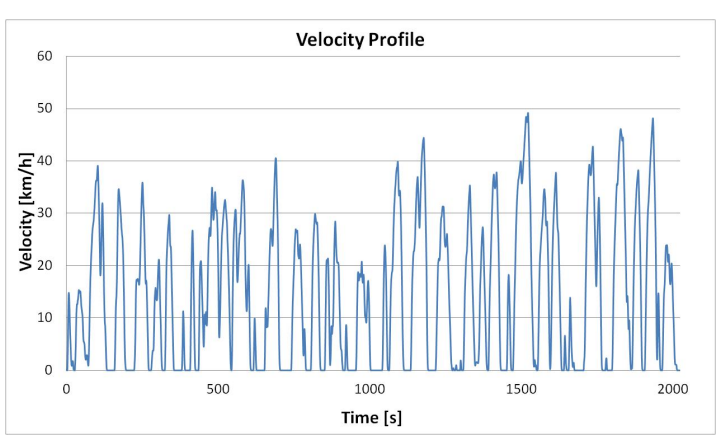

Figure 3: Drive cycle profile of line 27.

The driving cycle is based on line 27 of the Urban Transportation Company of Madrid, which in this case, it lasted 2023 seconds in a distance of 8.1 kilometres.

\subsection{Elevation profile}

It also has been considered the elevation profile. This a relevant parameter because it affects the driving performance and behaviour of the vehicle, especially in the torque, the current and the voltage that the electric drive motor provides in the cycle.

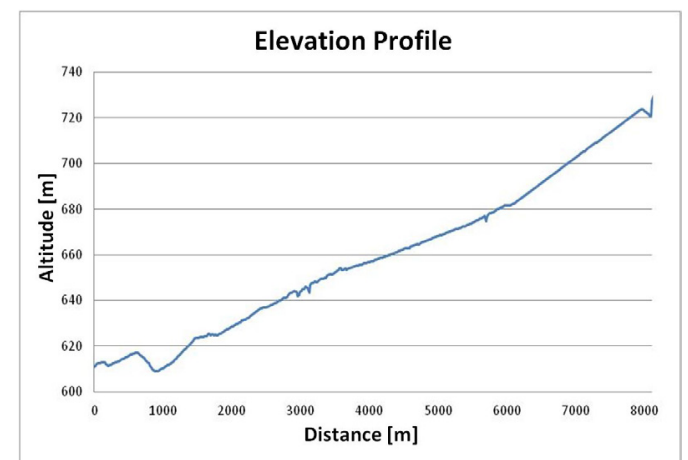

Figure 4: Elevation profile of the driving cycle (27 bus line) in the city of Madrid.

As it can be seen in figure 4, altitude over the sea level was considered. It also can be seen that the profile has a significant elevation of 100 meters.

\subsection{Driver behaviour}

Since the simulation has to obtain the driving performance of the vehicle, the driver behaviour was considered. This is due the calculation method used by the software, requires the driving controller in which this case, is referred to the driver. 
Driver behaviour is defined as a PID controller. Predefined standard driver parameters were chosen for performing the simulation.

\section{Controllers}

The controller programming determine the operating modes of the hybrid vehicle. As it was stated before, three controllers where programmed to achieve the operating modes of the vehicle in given circumstances of the driving cycle.

\subsection{Brake controller}

This controller was programmed to determine the instant when the brake pedal is applied by the driver. As when the vehicle is braking, the generated braking torque is transferred to the electric motor which it allows an electric brake with the electric motor (same as an engine brake) [2]. This is made to avoid a full mechanical braking process (by the callipers in the brake disks). At the same time, when the vehicle is first braking with the electric motor, the controller measures the vehicle speed and the commanded braking pressure from the driver, to convert the negative braking torque into the proper pressure for the brakes to slow down or stop the vehicle.

\subsection{Electric drive controller}

Same as the brake controller, the electric drive controller determines the instant in which the vehicle is in the braking process to allow the electric motor to operate as a generator. When the negative torque is provided by the rear wheels of the bus, it transfers to the electric motor in order to generate energy and store it in the battery pack.

In other words, this controller allows the electric motor to perform as an electric drive machine or as a generator, according to the driver behaviour in which depends if the acceleration or brake pedal is being used.

\subsection{Hybrid mode controller}

In the hybrid mode controller, the control strategy was programmed for allowing the efficiency and low emission of the vehicle. Control strategy is a rule o pattern that is present in the controller for hybrid and range extender operation. With the rest of the controllers, they commute the operation of every component so the vehicle can perform as a hybrid. The control strategy also takes in consideration the kind of cycle that the hybrid vehicle develops. In this case, a hybrid urban bus runs through a long route with several stops and accelerations with a average velocity of $12 \mathrm{~km} / \mathrm{h}$. The required power and energy for moving the vehicle does not only rely on the one supplied by the battery, but from the engine-generator set as well. For the case of an urban cycle in which there is a lot of stops and accelerations, the engine-generator set has to produce enough power to charge the battery, due that most of the cycle will be made in electric mode.

\section{Measurements. Real cycle data collecting.}

Real life measurements, in which the simulation is based, took place in Madrid. The tests were developed in the in-series hybrid bus modelled at this paper, with data collecting equipment onboard and in real cycle utilization.

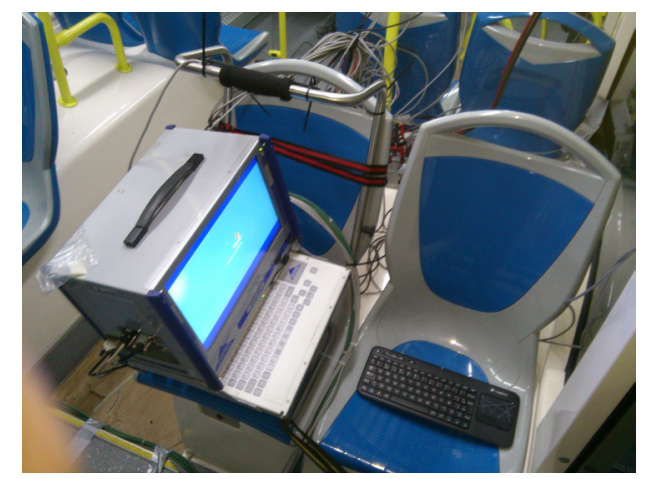

Figure 7: Measuring equipment employed in the full size hybrid urban bus tests.

\subsection{Equipment.}

All measures, except those corresponding to certain auxiliary equipment, was mad through data CAN Bus of the vehicle. PCAN Explorer ${ }^{\circledR}$ and a PC was used to collect the data involved.

To collect those data not present at the CAN Bus, it was used a Dewetron based analog-digital data logger, with current amps and voltage sensors.

To get the data regarding position and altitude, the vehicles was equipped with a GPS module. 


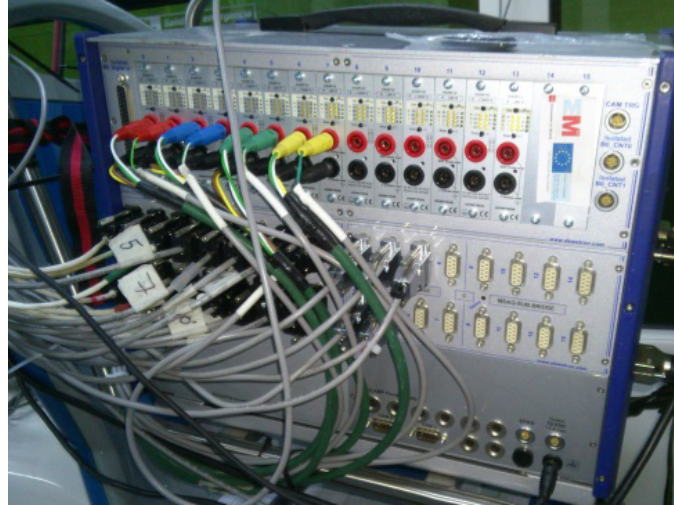

Figure 8: Data collection ports between the measuring equipment and the CAN Bus of the vehicle.

\subsection{Cycle}

\subsubsection{Bus lines}

All measurements have taken place on lines 27 and C1 of urban city route in Madrid (Figure 9) in collaboration with the Urban Transportation Company of the city. The driver was provided by this company and performed all tests.

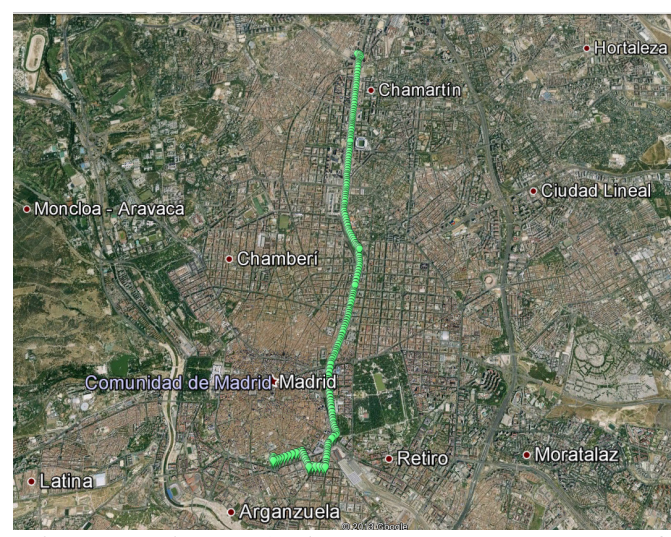

Figure 9: Line 27 (Urban Transport Company of Madrid) employed to the real tests. GPS data. Image: Google Earth

\section{Results and comparison}

A full performance simulation was made in order to evaluate the accuracy of the model by comparing the simulation run results with the data obtained on board the real size hybrid bus.

\subsection{Fuel consumption results}

Fuel consumption was taken into account and Table 1 shows the results.
Table 1. Comparison between fuel and $\mathrm{CO}_{2}$ emissions results.

\begin{tabular}{|c|l|l|}
\hline \multirow{2}{*}{$\begin{array}{c}\text { Real cycle } \\
\text { measurements }\end{array}$} & Fuel $[\mathrm{g} / \mathrm{km}]$ & 319.42 \\
\cline { 2 - 3 } $\begin{array}{c}\text { AVL model } \\
\text { results }\end{array}$ & $\mathrm{Fuel}[\mathrm{g} / \mathrm{gm} / \mathrm{km}]$ & 1008.24 \\
\cline { 2 - 3 } & $\mathrm{CO}_{2}[\mathrm{~g} / \mathrm{km}]$ & 303.62 \\
\hline
\end{tabular}

These results are quite accurate, with about $5 \%$ of average error. Differences at the results are probably as result of environment boundaries not taken into account or some electronic component efficiency a little increased.

\subsection{Driving behaviour results and comparison}

In order to evaluate the behaviour of the bus in the driving cycle, the next parameters were chosen:

- Electric motor speed.

- Electric motor torque.

- Mechanical power of the electric motor.

- Current.

- Voltage.

These parameters has a significant relevance because determines the performance of the powertrain of the hybrid vehicle [1]. Also, these were the main parameters that were acquired in the real test with the full size hybrid urban bus.

\subsubsection{Electric Motor Drive}

The electric motor behaviour compares de speed (in $\mathrm{rpm}$ ) and torque (in $\mathrm{Nm}$ ) generated in both situations (real test and simulation). Figure 10 shows a portion of the cycle for better evaluation and analysis while figure 11 shows the values obtained in the whole drive cycle.

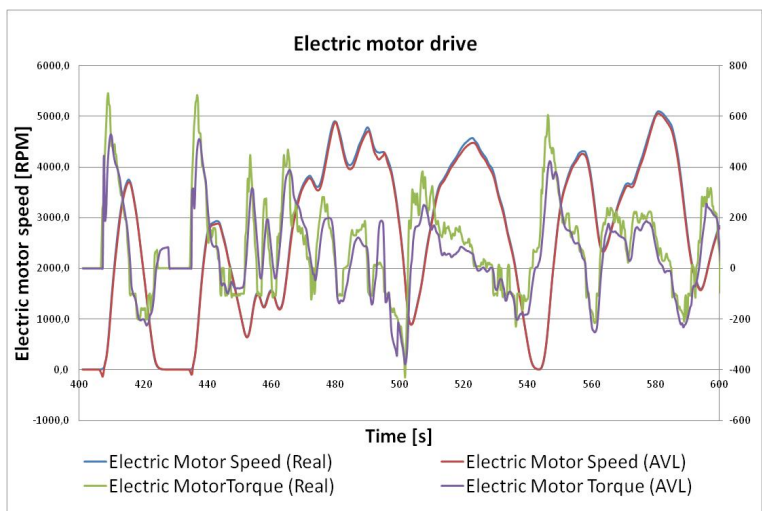

Figure 10: Portion of the cycle showing the electric motor drive behaviour. 


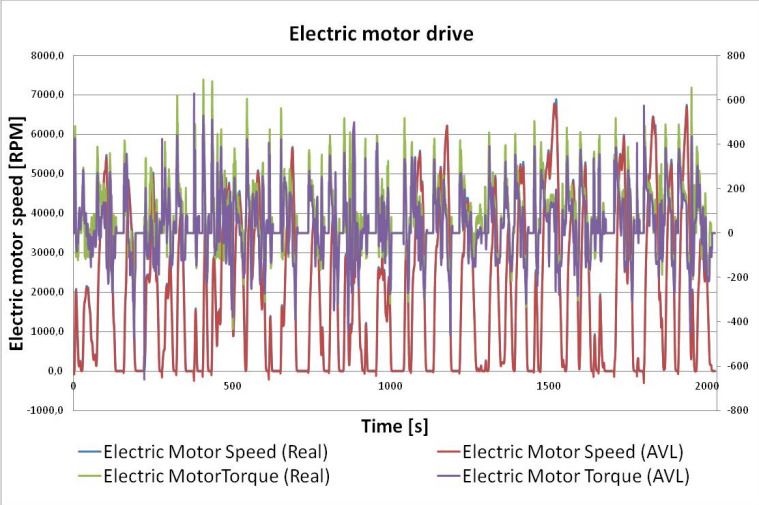

Figure 11: Electric motor drive comparison in the whole drive cycle.

As can be seen in figures 10 and 11, the accuracy of the model results regarding to motor speed and compared with real ones is quite good. The blue curve describes the speed that the electric motor developed in the real test, while the red line, describes the speed of the electric motor in the simulation. In the other hand, the torque obtained in the simulation, describes a similar pattern of the real test. The torque developed by the electric motor in the real test, is shown by the green curve, while the torque of the electric motor in the simulation is shown by the purple curve. Although it does not exactly match as the speed, simulated values of the torque are in a very close range and perform in a coherent set of scales.

\subsubsection{Mechanical power of the electric motor}

The mechanical power delivered by the electric motor in both situations (real test and simulation), is compared in the same way as the electric motor drive. Figure 12 shows these results in a portion of the cycle for better evaluation and analysis while figure 13 shows the mechanical power comparison in the whole drive cycle (in $\mathrm{kW}$ ).

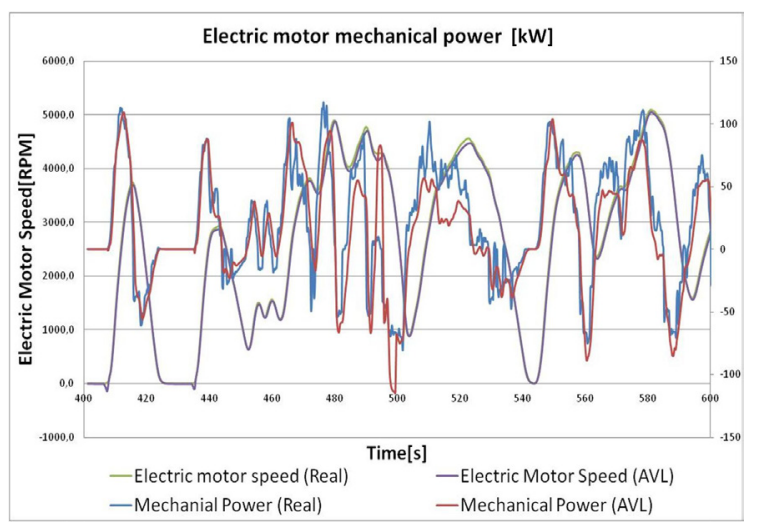

Figure 12: Portion of the cycle showing the mechanical power of the electric motor.

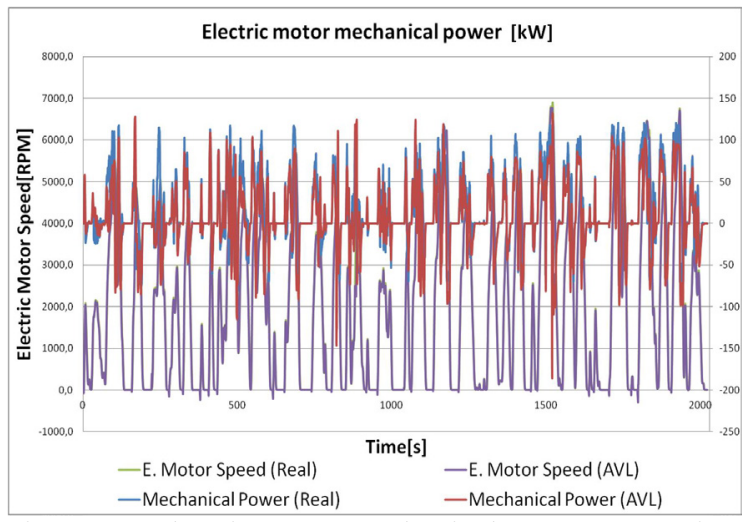

Figure 13: Electric motor mechanical power comparison in the whole driving cycle.

In the same way as the torque does, the mechanical power delivered by the electric machine tends to have slight variations in the comparison as well. In figures 12 and 13, the green and blue curves describes a comparison of the speed that the electric motor delivered in both conditions, while the blue curve describes the generated power by the electric motor in the real test and the red curve describes the power of the electric motor in the simulation. Also, it can be seen the values obtained in the simulation are in a close range of those obtained in real conditions and they perform in a coherent set of scales as well as the torque does.

\subsubsection{Current}

For a hybrid vehicle in which the power is supplied by an electric motor, it's of high relevance to analyse the current it delivered in the cycle. Current is an essential parameter that determines electrical power. In the same way then the previous results, figure 14 shows the current comparison in both situations (real test and simulation) in a portion of the cycle for better evaluation and analysis, while figure 15 shows the current comparison in the whole driving cycle (in Amps). 


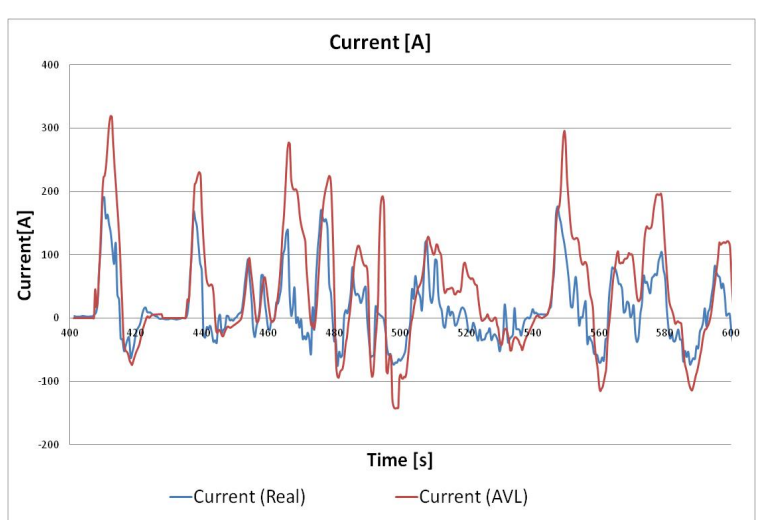

Figure 14: Current comparison in a portion of the cycle.

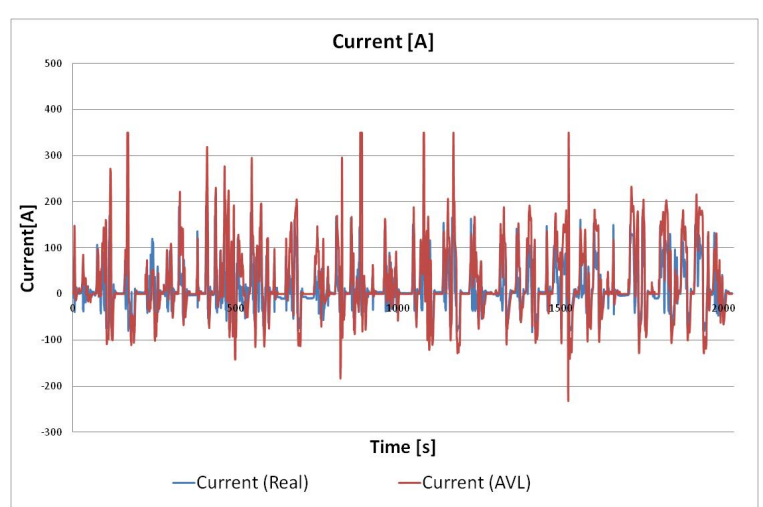

Figure 15: Current comparison in the whole cycle.

From all the results obtained in the simulation, the current is the one that shows more variation range than the rest of the results. Nevertheless, as it's expected, the current tends to draw the same pattern as the one described in real conditions and perform in a coherent set of values. In figures 14 and 15 , the blue curve describes the generated current in real conditions, while the red curve describes the current obtained in the simulation. Thanks to the BMS module in the model, the current has an expected behavior to prevent damaging the battery.

\subsubsection{Voltage}

The voltage is the last of the parameter to evaluate due that it has direct influence in the power and performance of the electric motor as well as the current do. In the same way than the previous results, figure 16 shows the voltage obtained in both situations (real test and simulation), while figure 17 shows the voltage obtained in the whole driving cycle (in Volts).

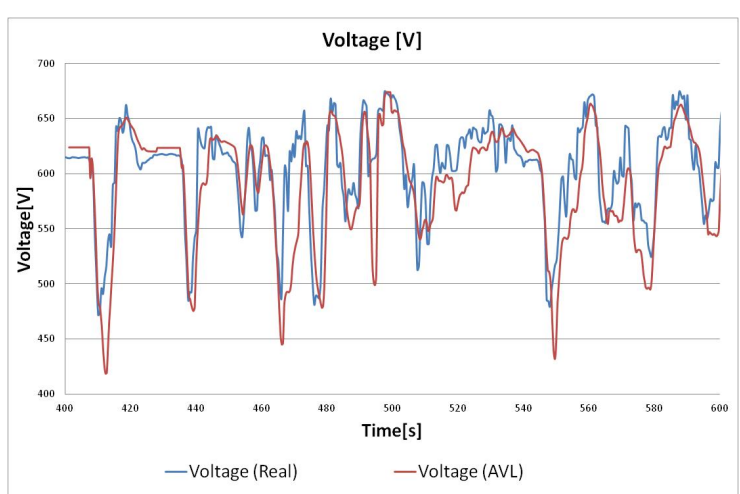

Figure 16: Voltage comparison in a portion of the cycle.

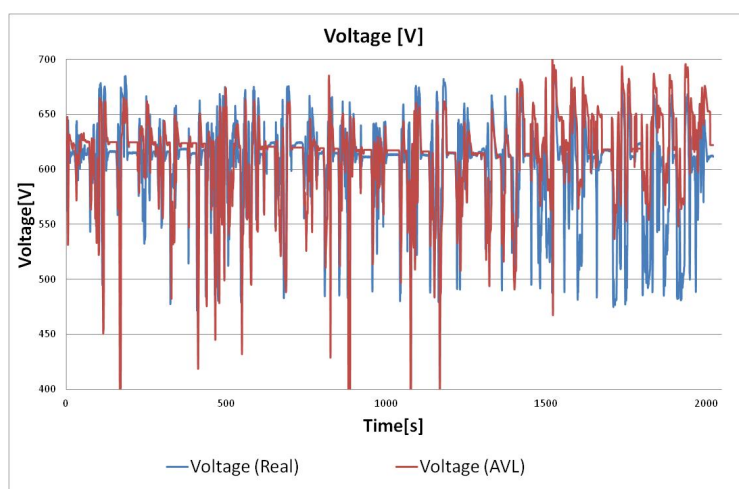

Figure 17: Voltage comparison in the whole cycle.

As it is expected, the voltage obtained in the simulation describes the same pattern of the voltage obtained in the real test and performs in a coherent set of values. In figures 16 and 17, The blue curve represents the voltage obtained in the real test, while the red curve represents the voltage obtained in the simulation.

\section{Conclusions}

The software model developed results show a high accuracy and very good harmony in all tests. A good synchrony between performance and efficiency/fuel results give to the model a solid foundation.

The implementation of hybrid urban buses in big cities represents a mayor breakthrough to reduce emissions and fuel consumption.

Due to results obtained in the simulation are very close to those measured in the real test, the model is reliable enough for further simulations with diverse routes described by other bus lines. That could be useful to analyse potential usages of those vehicles and to estimate their LCA. 
Also, it can be useful for improve strategy controls or to analyse the impact of changing components before doing it. Of course, it could be a great opportunity for the manufacturers, due to the reduced cost of modelling instead of test on-board.

\section{Acknowledgments}

We have to thank several institutions that have made possible this work, because of its contribution and collaboration.

- EMT Madrid: Urban Transport Company of Madrid, because of its collaboration on real cycle measurements.

- AVL ${ }^{\circledR}$ because of providing several licenses of its simulation software and its support with it. 


\section{References}

[1] Ben H. Thacker. Concepts of Model Verification and Validation. LA-14167-MS. NISA. Issued: October 2004.

[2] FarhadSangtarash et al. Effect of Different Regenerative Braking Strategies on Braking Performance and Fuel Economy in a Hybrid Electric Bus Employing CRUISE Vehicle Simulation, SAE International, 2008.

[3] Hans-Hermann Braess et al. Handbook of Automotive Engineering, ISBN 0-76800783-6, USA, SAE International, 2005

[4] Iqbal Husain. Electric and Hybrid Vehicles: Design Fundamentals, ISBN 978-1-43981175-7, USA, CRC Press, 2011

[5] John M. Miller. Propulsion Systems for Hybrid Vehicles. ISBN 0-86341-336-6, London, The Institution of Electrical Engineers, 2004

[6] José María López. El medio ambiente y el automóvil, ISBN 978-84-96437-70-8. Madrid, CIE Dossat, 2007

[7] Lino Guzzella et al. Vehicle Propulsion Systems: introduction to Modeling and Optimization, ISBN 978-3-540-74691-1, Zürich, Springer, 2007

[8] MehrdadEhsani et al. Modern Electric, Hybrid Electric and Fuel Cell Vehicles, ISBN 978-1-4200-5398-2, USA, CRC Press 2010

[9] Muhammad H. Rashid. Power Electronics Handbook, ISBN 978-0-12-088479-7, USA, Elsevier, 2007

[10] Regulation (EC) No 595/2009 of the European Parliament and of the Council of 18 June 2009 on type-approval of motor vehicles and engines with respect to emissions from heavy duty vehicles (Euro VI) and on access to vehicle repair and maintenance information

[11] Wang Baohua et al. Simulation on Performance of City Bus Based on Typical Urban Driving Cycle, Journal of Hubei Automotive Industries Institute; 2007. 


\section{Authors}

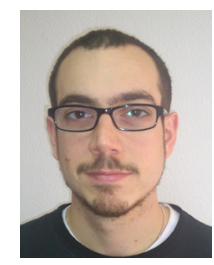

Daniel Resino Zamora is a Mechanical Engineer graduated at Technical University of Madrid. His professional experience covers different areas of transport. Nowadays, he is working for more than 3 years on Environmental and Powertrain Unit of Automotive Research Institute (INSIA) coordinating all technical areas of HEV projects. He is also attending to Automotive Engineering Master degree at INSIA, as well as starting his $\mathrm{PhD}$ studies on this area. He is author of other publications regarding to HEV heavy duty vehicles, like "Energetic requirements of heavy duty urban transport hybrid vehicles. Analysis of urban bus and waste collect cases in terms of use and electrification degree".

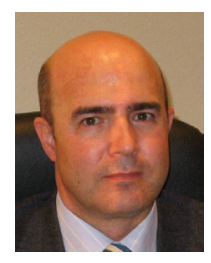

Dr. José María López is an Industrial $\mathrm{PhD}$, Deputy Director of INSIA (Automotive Research Institute) and Director of its Environmental and Powertrain Unit. He is also a Professor at Technical University of Madrid. $\mathrm{He}$ has extensive research experience in the field of vehicle propulsion systems and emissions due to road traffic, as well as guarantees their publications and conference papers on these topics. His research focuses on newer model development of propulsion systems for hybrid and electric vehicles and vehicle integration and validation. As an example, citing the design and development of a tri-hybrid powertrain with engine, fuel cell and solar panels for a lightweight urban electric vehicle, called EPISOL. He has published a book "The environment and the car. vehicle car challenge facing global $\mathrm{CO} 2$ reduction ", and recently coordinated the monograph" hybrid and electric vehicles" where relevant brands have participated
(HONDA, TOYOTA, Mistsubishi, GM-OPEL, etc.). Professor of several Masters and Coordinator of Master of Engineering Hybrid and Electric Vehicle of the UPM.

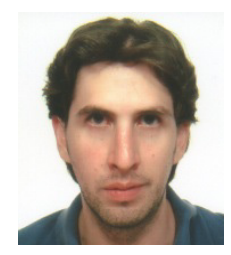

Carlos Julio Loboguerrero Carrasco is a Mechanical Egineer graduated at Universidad Iberoamericana in Mexico City. As today, he is doing the research Project for the master in mechanical engineering in the Technical University of Madrid. Prior to the current master program, he has acquired the master degree in Automotive Engineering at the Automotive Research Institute of the Technical University of Madrid, making him a professional and a person with enough knowledge in the automotive sector.

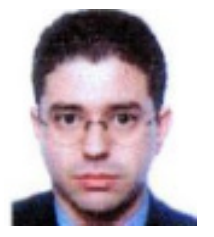

Juan José Delgado Vaca is a Mechanical Engineer graduated at Technical University of Madrid, specialized in internal combustion engines and its electronic control systems and architecture. His professional experience come out from two worldwide leader manufacturer companies of internal combustion engines. The scope of his experience covers aspects such as mechanical optimization, calibration adjustment-design and ECU networking. At the present time holding $\mathrm{PhD}$, particularly in the field of heavy duty hybrid vehicles control algorithms, optimization and validation. 